\title{
Classification of the Real Roots of the Quartic Equation and their Pythagorean Tunes
}

\author{
Emil M. Prodanov ${ }^{1}$ iD
}

Accepted: 15 September 2021 / Published online: 12 October 2021

(c) The Author(s) 2021

\begin{abstract}
Presented is a very detailed two-tier analysis of the location of the real roots of the general quartic equation $x^{4}+a x^{3}+b x^{2}+c x+d=0$ with real coefficients and the classification of the roots in terms of $a, b, c$, and $d$, without using any numerical approximations. Associated with the general quartic, there is a number of subsidiary quadratic equations (resolvent quadratic equations) whose roots allow this systematization as well as the determination of the bounds of the individual roots of the quartic. In many cases the root isolation intervals are found. The second tier of the analysis uses two subsidiary cubic equations (auxiliary cubic equations) and solving these, together with some of the resolvent quadratic equations, allows the full classification of the roots of the general quartic and also the determination of the isolation interval of each root. These isolation intervals involve the stationary points of the quartic (among others) and, by solving some of the resolvent quadratic equations, the isolation intervals of the stationary points of the quartic are also determined. The presented classification of the roots of the quartic equation is particularly useful in situations in which the equation stems from a model the coefficients of which are (functions of) the model parameters and solving cubic equations, let alone using the explicit quartic formulæ, is a daunting task. The only benefit in such cases would be to gain insight into the location of the roots and the proposed method provides this. Each possible case has been carefully studied and illustrated with a detailed figure containing a description of its specific characteristics, analysis based on solving cubic equations and analysis based on solving quadratic equations only. As the analysis of the roots of the quartic equation is done by studying the intersection points of the "sub-quartic" $x^{4}+a x^{3}+b x^{2}$ with a set of suitable parallel lines, a beautiful Pythagorean analogy can be found between these intersection points and the set of parallel lines on one hand and the musical notes and the staves representing different musical pitches on the other: each particular case of the quartic equation has its own short tune.
\end{abstract}

Keywords Quartic equation · Cubic equation · Quadratic equation · Roots · Isolation intervals $\cdot$ Pythagorean music

Mathematics Subject Classification $12 \mathrm{D} 10 \cdot 26 \mathrm{C} 10 \cdot 11 \mathrm{D} 41$

Emil M. Prodanov

emil.prodanov@tudublin.ie

1 School of Mathematical Sciences, Technological University Dublin, Park House, Grangegorman, 191

North circular Road, Dublin D07 EWV4, Ireland 


\section{Introduction}

The first book published in Europe that gives the complete solution to the quadratic equation was Liber Embadorum, written by Abraham bar Hiyya Ha-Nasi (latinized as Savasorda) in 1145. Savasorda was the first who translated and introduced Arabic algebra into Europe and this text became principal textbook for mathematical education. The first recorded mention of quartic equations is in Luca Pacioli's Summa de Arithmetica, Geometria, Proportioni e Proportionalità from 1494 (see [1] for a modern translation). Later in the Renaissance, Girolamo Cardano published a milestone in the history of mathematics-Artis Magna Sive de Regulis Algebraicis (1545) — a most comprehensive and systematic treatment of cubic and quartic equations. Cardano's masterpiece reflects the geometrical way of thinking at the time (numbers had to be positive as they represented lengths, areas, etc.) and avoids the use of negative numbers. The notation used is pre-Viète-for example, cup p: 6 reb aequalis 20 means $x^{3}+6 x=20$. For modern-day translation and notation, see [2].

Quartic equations have been observed all around for centuries. They appear in all branches of science. For example, in optics, the dispersion relationship is a quartic equation [3]. The points of intersection of two conic sections with given foci and directrices are, in general, the roots of a quartic equation.

The process of solving the general quartic equation

$$
x^{4}+a x^{3}+b x^{2}+c x+d=0
$$

involves the removal of the cubic term by using the substitution $x=y-a / 4$. This results in the depressed quartic $y^{4}+p y^{2}+q y+r=0$, where $p=b-3 a^{2} / 8, q=c+a^{3} / 8-a b / 2$, and $r=d-3 a^{4} / 256+a^{2} b / 16-a c / 4$. There are several algorithms for solving the depressed quartic equation and each of these involves solving a cubic equation called resolvent. The resolvents are different for the different algorithms. Finding the roots of the original quartic equation, once the roots of the resolvent cubic are known, is a straightforward procedure in each algorithm.

In the literature, the term root classification refers to the provision of all possible cases of the polynomial roots and it consists of a list of the multiplicities of all roots. On the other hand, the complete root classification of parametric polynomials consists of the root classification, together with the conditions which the equation coefficients should satisfy for each case of the root classification. Root classification and complete root classification have been extensively studied-see [4-8] and the references therein.

The complete root classification for the depressed quartic equation $x^{4}+p x^{2}+q x+r=0$ was found by Arnon in [4] and is as follows. Introducing:

$$
\begin{aligned}
& \delta(p, q, r)=256 r^{3}-128 p^{2} r^{2}+144 p q^{2} r+16 p^{4} r-27 q^{4}-4 p^{3} q^{2}, \\
& L(p, q, r)=8 p r-9 q^{2}-2 p^{3},
\end{aligned}
$$

one has:

$$
\begin{array}{ll}
1 & \delta>0 \wedge L>0 \wedge p<0 \\
2 & \delta=0 \wedge L>0 \wedge p<0 \\
3 & \delta=0 \wedge L=0 \wedge p<0 \wedge q \neq 0 \\
4 & \delta=0 \wedge L=0 \wedge p<0 \wedge q=0 \\
5 & \delta<0 \\
6 & \delta=0 \wedge L=0 \wedge p=0 \\
7 & \delta=0 \wedge L<0 \\
8 & {[\delta>0 \wedge(L \leq 0 \vee p>0)] \vee(\delta=0 \wedge L=0 \wedge p=0)}
\end{array}
$$


As can be seen, the root classification and the complete root classification do not deal with the location of the roots of the polynomial while the present work aims at addressing this issue. This paper introduces a different connotation of the term root classification by adding a new dimension to it. Using the methods presented in [9], a two-tier analysis of the location of the real roots of the general quartic equation (1) with real coefficients is presented and the root classification, in the sense of root multiplicities, conditions and, most importantly, root isolation or clustering intervals, is given. Namely, a technique is offered with which the precise isolation or clustering intervals of the roots of the parametric quartic equation are determined. The endpoints of these intervals are found as roots of quadratic equations with coefficients which are, at worst, integer multiples of the coefficients of the original quartic equation (cubic equations too, for the determination of the precise isolation interval of each root). The proposed classification of the roots of the quartic equation-in terms of the coefficients of the equation and based on solving lower-degree equations-is particularly useful when the quartic stems from the study of some model and the coefficients of the resulting equation are (functions of) the model parameters. Even the presence of a single parameter in the equation makes the application of the cubic formulæ, let alone the quartic formulæ, practically impossible and the only benefit would be to get insight into the location of the roots. The presence of parameters in the equation renders any approach, based on numerical methods, tedious and time consuming. Even the determination of whether a particular case is a casus irreducibilis is not possible.

Associated with each quartic, there is a number of subsidiary quadratic equations, referred to in this text as resolvent quadratic equations, with the help of which the roots of the general quartic are systematized in terms of its coefficients $a, b, c$, and $d$. Additionally, the bounds on individual roots are determined. In many cases the root isolation intervals are found, but there is some residual indeterminacy as some intervals may contain either two roots of the quartic or no roots at all. The individual root bounds themselves are associated with the roots of $x^{2}+a x+b=0$ and $c x+d=0$, or with the non-zero stationary points of $x^{2}\left(x^{2}+a x+b\right)$, if real roots are absent, or with the points of curvature change of $x^{2}\left(x^{2}+a x+b\right)$, if real roots and non-zero stationary points are absent, or with the point of vanishing third derivative of $x^{2}\left(x^{2}+a x+b\right)$, if real roots and non-zero stationary points, and curvature change points are absent.

The other tier of the presented analysis is the full classification of the roots of the quartic in terms of its coefficients and, also, the determination of the isolation interval of each root of the quartic by solving two cubic equations and some of the resolvent quadratic equations. These two cubic equations are subsidiary to each quartic and are referred to in this paper as auxiliary cubic equations - in order to make a distinction from the resolvent cubic equation.

It can be argued why it is necessary to address not one, but two cubic equations (the auxiliary cubic equations) which do not yield the roots of the quartic, but only reveal their isolation intervals, and what can be gained by doing so. Indeed, it suffices to solve a single cubic equation (the resolvent) in order to find the actual roots of any quartic. The answer to this is in the search for rules and patterns through abstraction to gain insight on how different coefficient vectors affect the roots. Systematization and having predictive powers are always a bonus and merit investigation. If analysis of the roots of the quartic could be done with equations of degree two, one would expect that using equations of degree three would be more informative, despite getting into the realm where the procedure of finding the roots through the explicit formulæ is applied. The analysis based on solving cubic equations complements the picture revealed by the analysis based on quadratic equations. The resulting systematization and classification are not possible if one addresses the explicit formulæ for the roots of the quartic - these are rather unwieldy and one cannot trace how the variation of 
a particular coefficient of the given quartic affects the roots, i.e. the coefficients of the quartic enter the root formulæ in an intricate combination involving the $\operatorname{root}(\mathrm{s})$ of the resolvent cubic (which, in turn, depend on the coefficients of the quartic) and one cannot discern the individual contribution of each coefficient of the quartic to the location of its roots. The proposed analysis based on cubic equations also has heuristic potential: just by observation of the coefficients and whether they fall into specific ranges, one can predict the number of real roots and also find their isolation intervals. For example, for any $a, c$, and $d$, when $b>3 a^{2} / 2$, the quartic cannot have four real roots. If, additionally, the free term $d$ is negative and $c$ is also negative, then the quartic has one negative root smaller than $-d / c$ and one positive root greater than the only real non-zero root $\lambda>0$ of the equation obtained from the quartic after removing the free term $d$. If $\mu$ denotes the only stationary point of quartic ( $\mu>0$ in this case) and $d$ is positive and greater than $\mu^{4}+a \mu^{3}+b \mu^{2}+c \mu$ (which itself is positive), then the quartic has no real roots. If $0<d<\mu^{4}+a \mu^{3}+b \mu^{2}+c \mu$, then the quartic has two positive roots - one bigger than $-d / c$ and smaller than $\mu$, the other-bigger than $\mu$ and smaller than $\lambda$.

In the presented analysis, the isolation intervals of the stationary points of the general quartic are also determined-with the help of the resolvent quadratic equations.

Every possible case has been individually studied and illustrated with a detailed figure containing a description of its specific characteristics, analysis based on solving cubic equations and analysis based on solving quadratic equations only.

Pythagoras believed that numbers hold the key to understanding the Cosmos. He thought that mathematics could explain everything, including music. Pythagoras perceived the inherent properties of mathematics within music and his quest to relate harmony to proportion was taken up by the School of Plato. In this paper, the analysis of the roots of the quartic equation is done after studying the intersection points of the "sub-quartic" $x^{4}+a x^{3}+b x^{2}$ with a set of suitable parallel lines. A beautiful Pythagorean analogy exists between these intersection points and the set of parallel lines on one hand and the musical notes and the staves representing different musical pitches on the other. Even more, each case of the quartic equation has its own tune.

\section{Subsidiary Cubic, Quadratic, and Linear Equations}

Each quartic is associated with a number of subsidiary cubic, quadratic, and linear equations whose roots can be used for the classification of the roots of the quartic. All figures are given in Online Supplementary Material.

In parallel with the presentation of the analysis, Figs. 1 to 5 illustrate, through a particular example with the quartic $x^{4}+x^{3}-3 x^{2}-x+1$, the full procedure of finding the isolation interval of each root of the quartic, based on solving cubic equations, and the localization of the roots by determination of the individual root bounds, based on solving quadratic equations only. The isolation intervals of the stationary points of this quartic are also found.

Figures 6 to 12 illustrate some patterns associated with the general quartic and these are used for the classification of the roots of the quartic.

Taking the free term $d$ of the quartic and varying it, yields a one-parameter congruence of quartics, all having the same set of stationary points (which are either three- - two local minima and a local maximum or a saddle point and a local minimum, or just one-a minimum). Let $\mu_{i}$ denote the stationary point(s). For each $\mu_{i}$, there is a "special" quartic within this congruence: $x^{4}+a x^{3}+b x^{2}+c x+\delta_{i}$ - the one whose graph is tangent to the abscissa at that particular 
stationary point. The derivative of the "special" quartic is also zero at this stationary point, namely, for $x^{4}+a x^{3}+b x^{2}+c x+\delta_{i}$ the stationary point $\mu_{i}$ is also a double root [or a triple root, if the original quartic has a saddle at $\mu_{i}$, or a quadruple root $-a / 4$ in the case of the quartic $(x-a / 4)^{4}=x^{4}+a x^{3}+(3 / 8) a^{2} x^{2}+(1 / 16) a^{3} x+(1 / 256) a^{4}$, which coincides with its only "special" quartic]. Setting the derivative of the quartic equal to zero yields the set of its stationary points and the resulting equation,

$$
4 x^{3}+3 a x^{2}+2 b x+c=0,
$$

is referred to in this text as first auxiliary cubic equation.

Substituting each real root $\mu_{i}$ of this equation into the corresponding "special" quartic equation $x^{4}+a x^{3}+b x^{2}+c x+\delta_{i}=0$, immediately gives:

$$
\delta_{i}=-\mu_{i}^{4}-a \mu_{i}^{3}-b \mu_{i}^{2}-c \mu_{i} .
$$

Thus the "special" quartics are given by $x^{4}-\mu_{i}^{4}+a\left(x^{3}-\mu_{i}^{3}\right)+b\left(x^{2}-\mu_{i}^{2}\right)+c\left(x-\mu_{i}\right)$.

The discriminant of the first auxiliary cubic equation is $\Delta_{1}=-432 c^{2}-432 a\left(a^{2} / 4-\right.$ $b) c+128 b^{2}\left[(9 / 8) a^{2} / 4-b\right]$. It can be viewed as a quadratic in $c$, treated as unknown, with $a$ and $b$ treated as parameters. The first resolvent quadratic equation is obtained by setting $\Delta_{1}=0$ :

$$
c^{2}+a\left(\frac{a^{2}}{4}-b\right) c-\frac{8}{27} b^{2}\left(\frac{9}{8} \frac{a^{2}}{4}-b\right)=0 .
$$

The roots of this equation,

$$
c_{1,2}(a, b)=c_{0} \pm \frac{2 \sqrt{6}}{9} \sqrt{\left(\frac{3}{2} \frac{a^{2}}{4}-b\right)^{3}}, \quad \text { with } \quad c_{0}(a, b)=\frac{1}{2} a\left(b-\frac{a^{2}}{4}\right)
$$

play a very important role in the analysis. For any given quartic, one has to see first whether the coefficient $c$ of the linear term falls between the roots $c_{1,2}(a, b)$ or outside them. If $c_{2}(a, b) \leq c \leq c_{1}(a, b)$, then the discriminant $\Delta_{1}$ is positive and the quartic has three stationary points: $\mu_{1,2,3}$. Otherwise it has just one: $\mu_{1}$. In the first case, the quartic can have either 0 , or 2 , or 4 real roots; in the second case it can have either 0 or 2 real roots. It is immediately obvious that, for any $a, c$, and $d$, when $b>(3 / 2)\left(a^{2} / 4\right)$, the quartic can have either 0 or 2 real roots only (the first resolvent quadratic equation (6) has negative discriminant).

When the discriminant $\Delta_{1}$ of the first auxiliary cubic equation (4) is equal to zero, that is, when $c=c_{1,2}(a, b)$, the original quartic with $c$ replaced by $c_{1,2}$ has a saddle point at $\eta_{1,2}$ and a local minimum at $\theta_{1,2}$. The corresponding "special" quartic at $\eta_{1,2}$ is $x^{4}+a x^{3}+b x^{2}+$ $c_{1,2} x+d_{1,2}$, where $d_{1,2}=-\eta_{1,2}^{4}-a \eta_{1,2}^{3}-b \eta_{1,2}^{2}-c_{1,2} \eta_{1,2}$, and for the "special" quartic, $\eta_{1,2}$ is a triple root. The points $\eta_{1,2}$ and $\theta_{1,2}$ can be easily found as the "special" quartic $x^{4}+a x^{3}+b x^{2}+c_{1,2} x+d_{1,2}$, its first derivative, and its second derivative are all zero at $\eta_{1,2}$. In other words, one has to start with solving the second resolvent quadratic equation,

$$
6 x^{2}+3 a x+b=0
$$

the roots of which are

$$
\eta_{1,2}=-\frac{1}{4} a \pm \frac{\sqrt{6}}{6} \sqrt{\frac{3}{2} \frac{a^{2}}{4}-b}
$$


then write down the vanishing first derivative of the ("special") quartic as $4 x^{3}+3 a x^{2}+2 b x+$ $c_{1,2}=4\left(x-\eta_{1,2}\right)^{2}\left(x-\theta_{1,2}\right)$, and then compare the coefficients of the quadratic terms. This will give $\theta_{1,2}=-(3 / 4) a-2 \eta_{1,2}$ and hence:

$$
\theta_{1,2}=-\frac{1}{4} a \pm \frac{\sqrt{6}}{3} \sqrt{\frac{3}{2} \frac{a^{2}}{4}-b} .
$$

One could observe that $-a / 4$ is the quadruple root of the quartic $x^{4}+a x^{3}+(3 / 8) a^{2} x^{2}+$ $(1 / 16) a^{3} x+(1 / 256) a^{4}=0$.

With the help of $\eta_{1,2}$ and $\theta_{1,2}$, the isolation intervals of the stationary points $\mu_{i}$ of the general quartic can be easily found (see the example on Figure 6 in the Online Supplementary Material). In the regime of increasing $c$ and starting with $c<c_{2}$, there is only one stationary point (local minimum) at $\widehat{\mu}_{1}>\theta_{2}$. When $c=c_{2}$, the quartic has a saddle point at $\eta_{2}$ and a local minimum at $\theta_{2}$. As soon as $c$ gets bigger than $c_{2}$, the saddle point $\eta_{2}$ bifurcates into two stationary points $\mu_{2}$ and $\mu_{3}$ on either side of $\eta_{2}$ : a local maximum at $\mu_{2}$ such that $\eta_{2}<\mu_{2}<\eta_{1}$ and a local minimum at $\mu_{3}$ such that $\theta_{1}<\mu_{3}<\eta_{2}$. The local minimum $\tilde{\mu}_{1}$ remains as $\mu_{1}$ and is such that $\eta_{1}<\mu_{1}<\theta_{2}$. With the further increase of $c$, the local maximum at $\mu_{2}$ and the right local minimum (at $\mu_{1}$ ) get closer to each other and coalesce at $\eta_{1}$ when $c=c_{1}$. The left local minimum is then at $\theta_{1}$. This corresponds to a saddle point $\eta_{1}$ and a local minimum at $\theta_{1}$ for the quartic with $c=c_{1}$. When $c$ becomes bigger than $c_{1}$, the quartic will have only one stationary point - the left local minimum $\mu_{3}$ remains as $\tilde{\mu}_{1}<\theta_{1}$.

To summarize, the isolation intervals of the stationary points of the general quartic are as follows (dropping the tilde and the hat):

(i) If $c<c_{2}$, the quartic has a single local minimum $\mu_{1}>\theta_{2}$.

(ii) If $c=c_{2}$, the quartic has a saddle point at $\eta_{2}$ and a local minimum at $\theta_{2}$.

(iii) If $c_{2}<c<c_{1}$, the quartic has a local minimum at $\mu_{3}$ where $\theta_{1}<\mu_{3}<\eta_{2}$, a local maximum at $\mu_{2}$ where $\eta_{2}<\mu_{2}<\eta_{1}$, and local minimum at $\mu_{1}$ where $\eta_{1}<\mu_{1}<\theta_{2}$.

(iv) If $c=c_{1}$, the quartic has a saddle point at $\eta_{1}$ and a local minimum at $\theta_{1}$.

(v) If $c>c_{1}$, the quartic has a single local minimum $\mu_{1}<\theta_{1}$.

For the analysis further, one also needs to determine the other two roots $\xi_{1,2}^{(i)}$ of the "special" quartics $x^{4}+a x^{3}+b x^{2}+c x+\delta_{i}$ (recall that $\mu_{i}$ is at least a double root for them). One has:

$$
x^{4}+a x^{3}+b x^{2}+c x+\delta_{i}=\left(x-\mu_{i}\right)^{2}\left(x-\xi_{1}^{(i)}\right)\left(x-\xi_{2}^{(i)}\right)=0 .
$$

Viète formulæ give: $\xi_{1}^{(i)}+\xi_{2}^{(i)}=-a-2 \mu_{i}$ and $2 \mu_{i}\left[\xi_{1}^{(i)}+\xi_{2}^{(i)}\right]+\mu_{i}^{2}+\xi_{1}^{(i)} \xi_{2}^{(i)}=b$. From these one finds that $\xi_{1}^{(i)} \xi_{2}^{(i)}=b+3 \mu_{i}^{2}+2 a \mu_{i}$. If $x \neq \mu_{i}$ then (11) reduces to

$$
x^{2}+\left(a+2 \mu_{i}\right) x+b+3 \mu_{i}^{2}+2 a \mu_{i}=0 .
$$

This is the third resolvent quadratic equation. The roots of this equation are:

$$
\xi_{1,2}^{(i)}=-\frac{1}{2} a-\mu_{i} \pm \frac{1}{2} \sqrt{a^{2}-4 a \mu_{i}-8 \mu_{i}^{2}-4 b} .
$$

Note that the roots of the third resolvent quadratic equation (12) depend on the roots $\mu_{i}$ of the first auxiliary cubic equation (4). That is, to find the $\xi$ 's, one needs to find at least one of the stationary points of the quartic. Thus, the third resolvent quadratic equation should be used in the analysis based on solving cubic equations.

Separately, for one of the three "special" quartics $x^{4}+a x^{3}+b x^{2}+c x+\delta_{i}$, the roots $\xi_{1,2}^{(i)}$ of the third resolvent quadratic equation (12) are not real, while for the remaining two 
they are real (unless the original quartic has the same value at its two local minima, in which case two of the "special" quartics $x^{4}+a x^{3}+b x^{2}+c x+\delta_{i}$ coincide and so all "special" quartics will have four real roots) — see Figure 2 in the Online Supplementary Material.

In the congruence of quartics, there is another significant quartic-the one that passes through the origin-i.e. this is a privileged quartic as it is the only one that has zero as a root. It is obtained from the original quartic by removing the free term $d$. The remaining three roots of this privileged quartic are found by solving the second auxiliary cubic equation:

$$
x^{3}+a x^{2}+b x+c=0 .
$$

If the discriminant $\Delta_{2}=-27 c^{2}+\left(-4 a^{3}+18 a b\right) c+a^{2} b^{2}-4 b^{3}$ of this equation is negative, there is only one real root: $\lambda_{1}$. If it is not negative, there are three real roots: $\lambda_{0,1,2}$. To determine which of these occurs, set $\Delta_{2}=0$ to obtain the fourth resolvent quadratic equation:

$$
c^{2}+\frac{2}{3} a\left(\frac{8}{9} \frac{a^{2}}{4}-b\right) c-\frac{4}{27} b^{2}\left(\frac{1}{4} a^{2}-b\right)=0 .
$$

Once again, setting a discriminant of a cubic equal to zero is viewed as a quadratic in the unknown $c$ with $a$ and $b$ treated as parameters. The roots of this equation,

$$
\gamma_{1,2}(a, b)=\frac{1}{3} a\left(b-\frac{8}{9} \frac{a^{2}}{4}\right) \pm \frac{2 \sqrt{3}}{9} \sqrt{\left(\frac{4}{3} \frac{a^{2}}{4}-b\right)^{3}} .
$$

also play a very important role in the analysis. If the given $c$ is such that $\gamma_{2}(a, b) \leq c \leq$ $\gamma_{1}(a, b)$, then the quartic $x^{4}+a x^{3}+b x^{2}+c x$ has four real roots: 0 and $\lambda_{0,1,2}$ (there may be zeros among the $\lambda$ 's). Otherwise, the $x^{4}+a x^{3}+b x^{2}+c x$ has only two real roots: zero and $\lambda_{1}$ (which may also be zero).

Following the ideas of [9], the four "degrees of freedom" of the general quartic $x^{4}+$ $a x^{3}+b x^{2}+c x+d$ are split equally between two separate polynomials, $x^{4}+a x^{3}+b x^{2}$ and $-c x-d$, the difference of which comprises the given quartic and the "interaction" between which gives the roots of the quartic:

$$
x^{2}\left(x^{2}+a x+b\right)=-c x-d
$$

- see Figure 3 (and also Figures 4 and 5 in the Online Supplementary Material) where this is illustrated with an example.

It may be tempting to depress the quartic and analyse only its "three-dimensional projection" $y^{4}+p y^{2}+q y+r=0$, but by doing so, study of how the coefficients of the original quartic affect its roots would not be possible as these coefficients would be "dissolved" into $p, q$, and $r$.

It is quite easy to analyse the two parts of the "split" quartic and, hence, the quartic itselfone of the "components" is a straight line, while the other is a quadratic "in disguise"-in the sense that it is a quartic having zero as a double root and allowing analysis not more difficult than that of a genuine quadratic (with the possible addition of a pair of stationary points and/or a pair of curvature change points, and the addition of a point where the third derivative vanishes).

For any given $a$ and $b$, i.e. for any "sub-quartic" $x^{2}\left(x^{2}+a x+b\right)$, one can find a straight line $-c^{\dagger} x-d^{\dagger}$ such that it will be tangent to $x^{2}\left(x^{2}+a x+b\right)$ at two points, say $\alpha$ and $\beta$. This means that the obtained in this manner quartic, $x^{4}+a x^{3}+b x^{2}+c^{\dagger} x+d^{\dagger}$, has two double roots: $\alpha$ and $\beta$. That is, $x^{4}+a x^{3}+b x^{2}+c^{\dagger} x+d^{\dagger}=(x-\alpha)^{2}(x-\beta)^{2}=$ $x^{4}-2(\alpha+\beta) x^{3}+\left(\alpha^{2}+\beta^{2}+4 \alpha \beta\right) x^{2}-2 \alpha \beta(\alpha+\beta) x+\alpha^{2} \beta^{2}$. Comparing the corresponding coefficients yields: $c^{\dagger}=(1 / 2) a\left(b-a^{2} / 4\right)$. This is exactly equal to $c_{0}$-see the roots (7) of 
the first resolvent quadratic equation (6). One also gets $d^{\dagger}=(1 / 4)\left(b-a^{2} / 4\right)^{2}>0$. From now on, $c_{0}$ and $d_{0}$ will be used instead of $c^{\dagger}$ and $d^{\dagger}$.

The quartic $x^{4}+a x^{3}+b x^{2}+c_{0} x+d_{0}$ is very important for the classification of the roots of the general quartic. This is better visualized on the tablecloth of the "split" quartic (17). The determination of whether $c$ is smaller, equal, or greater than $c_{0}$ will determine the ordering of the $\delta$ 's and will also determine the number of intersection points between the "sub-quartic" $x^{2}\left(x^{2}+a x+b\right)$ and $-c x-d$ for any value of $d$. For example, on Figure 5 in the Online Supplementary Material, one has $0<c_{0}=-1.63<c=-1$. Thus, if one studies the intersection points of $x^{2}\left(x^{2}+a x+b\right)$ with $-c x-d$ in the regime of increasing $(-d)$ starting from $-\infty$, i.e. "sliding" a straight line with fixed slope $(-c)$ upwards, intersections of this straight line with $x^{2}\left(x^{2}+a x+b\right)$ will occur first in the third quadrant before they occur in the forth.

From the roots (7) of the first resolvent quadratic equation (6), it is immediately obvious that $c_{2}(a, b) \leq c_{0}(a, b) \leq c_{1}(a, b)$ for any $a$ and $b$. The graph of $c_{0}$ as a function of $a$ and $b$ is shown on Figure 7 in the Online Supplementary Material. Figures 8 to 11 in the Online Supplementary Material show that, for any $a$ and $b$, the following holds for the general quartic:

$$
c_{2}(a, b) \leq \gamma_{2}(a, b) \leq c_{0}(a, b) \leq \gamma_{1}(a, b) \leq c_{1}(a, b) .
$$

Depending on $a$ and $b$, the place of zero in the above chain of inequalities could be anywhere. For the analysis of the general quartic, the very first step is the determination of these numbers and the following step is to find the place of 0 and the given $c$ in the above. Figure 12 in the Online Supplementary Material shows this chain when $a$ and $b$ are both negative, in which case one has $c_{2}<\gamma_{2}<0<c_{0}<\gamma_{1}<c_{1}$. On Figure 12 in the Online Supplementary Material, the "separator" straight lines $-c_{1,2} x-d_{1,2},-\gamma_{1,2} x$, and $-c_{o} x-d_{0}$ are drawn and this clearly demonstrates that (including also the coordinate axes) there are seven ranges in which $c$ may fall. Each of these is individually studied. It has its own peculiarities that reflect on the number of roots and their localization.

Next, for the classification of the roots based on solving cubic equations, one needs to find the place of $(-d)$ among the $(-\delta)$ 's and zero-see Figure 4 in the Online Supplementary Material where this is illustrated with the quartic equation $x^{4}+x^{3}-3 x^{2}-x+1=0$. The role of the second auxiliary cubic equation (14) and its roots $\lambda_{0,1,2}$ now becomes clear. Keeping $a$ and $b$ fixed [i.e. not changing the "sub-quartic" $x^{2}\left(x^{2}+a x+b\right)$ ], and only varying $c$ would "move" the stationary points $\mu_{i}$ along the "sub-quartic" $x^{2}\left(x^{2}+a x+b\right)$. For the example with $a=1$ and $b=-3$ on Figures 1 to 5 in the Online Supplementary Material, for as long as $c<0$, one always has $-\delta_{2}>0$ for the "sub-quartic" $x^{2}\left(x^{2}+a x+b\right)$. On the other hand however, $-\delta_{1}$ and $-\delta_{3}$ could be anywhere. Because the second auxiliary cubic equation (14) has three real roots $\lambda_{0,1,2},-\delta_{1}$ and $-\delta_{3}$ are both negative-these are on the "other side" (opposite side of $-\delta_{2}$ ) of the straight line $-c x-0$ (the linear "part" of the privileged quartic $\left.x^{4}+a x^{3}+b x^{2}+c x\right)$. If the second auxiliary cubic equation (14) had just one root $\lambda_{1}$, then $-\delta_{1}$ and $-\delta_{3}$ would both be positive. And because $c>c_{0}$, one has $-\delta_{3}<-\delta_{1}$.

For the other tier of the analysis—based on solving quadratic equations only-one does not have the $\mu$ 's, the $\delta$ 's, and the $\lambda^{\prime}$ s explicitly. The isolation intervals of the $\delta$ 's and the $\lambda$ 's can be found in a manner similar to the one used for the determination of the isolation intervals of the $\mu$ 's or one can see [9] for the full classification of the roots of the cubic equation and the determination of the isolation intervals of its roots. The "separator" line $-c x$ can still be used without knowing the loci of its point(s) of intersection with the "sub-quartic" $x^{2}\left(x^{2}+a x+b\right)$, but knowing if these are three or just one. The "separator" lines $-c x-\delta_{i}$ 
can no longer be used for analysis based on solving quadratic equations only. There is a way however, to find "replacements".

The "sub-quartic" $x^{2}\left(x^{2}+a x+b\right)$ has zero as a double root and two more roots which are the roots of the fifth resolvent quadratic equation

$$
x^{2}+a x+b=0
$$

the roots of which are

$$
\rho_{1,2}=-\frac{1}{2} a \pm \sqrt{\frac{a^{2}}{4}-b} .
$$

These are real for $b \leq a^{2} / 4$.

Then, one takes the given $c$ and draws the two parallel lines with equations $-c\left(x-\rho_{1,2}\right)$. These straight lines are the sought "replacements" of the "separator" lines $-c x-\delta_{i}$ and their intersections with the ordinate-the "marker" points $c \rho_{1,2}$ - are the "replacements" of the $(-\delta)$ 's. This allows the analysis based on solving quadratic equations only to be performed in manner fully analogous to that of the analysis based on solving cubic equations-see Figure 5 in the Online Supplementary Material.

All possibilities for this analysis are shown on Figures 1.1 to 1.14 and 2.1 to 2.14 in the Online Supplementary Material.

If $b>a^{2} / 4$ ( $\rho_{1,2}$ not being real), a different pair of characteristic points of the "subquartic" $x^{2}\left(x^{2}+a x+b\right)$ should be chosen as "marker" points - the two non-zero critical points $\sigma_{1,2}$ of $x^{2}\left(x^{2}+a x+b\right)$. Clearly, recourse to these can be made for $a^{2} / 4<b \leq(9 / 8) a^{2} / 4-$ as can be easily seen from the sixth resolvent quadratic equation

$$
4 x^{2}+3 a x+2 b=0
$$

the roots of which are the non-zero critical points of $x^{2}\left(x^{2}+a x+b\right)$ given by

$$
\sigma_{h, H}=-\frac{3}{8} a \pm \frac{\sqrt{2}}{2} \sqrt{\frac{9}{8} \frac{a^{2}}{4}-b .}
$$

One then calculates the values $H$ and $h$ of $x^{2}\left(x^{2}+a x+b\right)$ at $\sigma_{H}$ and $\sigma_{h}$ respectively and draws the parallel lines $-c\left(x-\sigma_{H}\right)+H$ and $-c\left(x-\sigma_{h}\right)+h$ to serve as "separators". These intersect the ordinate at the "marker" points $c \sigma_{H}+H$ and $c \sigma_{h}+h$. One has to be careful because, depending on $c$, one can have zero, $c \sigma_{H}+H$, and $c \sigma_{h}+h$ in any order.

All possibilities for this analysis are shown on Figures 3.1 to 3.14 in the Online Supplementary Material.

Should $b$ be greater than $(9 / 8)\left(a^{2} / 4\right)$, then the "sub-quartic" $x^{2}\left(x^{2}+a x+b\right)$ will not have critical points. One should then use the points of curvature change [non-zero first derivative, but vanishing second derivative of the "sub-quartic" $\left.x^{2}\left(x^{2}+a x+b\right)\right]$. These are real for $b \leq(3 / 2)\left(a^{2} / 4\right)$. They are the roots of the second resolvent quadratic equation and on Figures 4.1 to 4.14 and, also 5.1 to 5.10 in the Online Supplementary Material (where the relevant analysis is), they are denoted by

$$
\tau_{h, H}=-\frac{1}{4} a \pm \frac{\sqrt{6}}{6} \sqrt{\frac{3}{2} \frac{a^{2}}{4}-b .}
$$

As with the critical points $\sigma_{1,2}$, one then calculates the values $H$ and $h$ of $x^{2}\left(x^{2}+a x+b\right)$ at $\tau_{H}$ and $\tau_{h}$ respectively and draws the parallel lines $-c\left(x-\tau_{H}\right)+H$ and $-c\left(x-\tau_{h}\right)+h$ to serve as "separators". These intersect the ordinate at the "marker" points $c \tau_{H}+H$ and 
$c \tau_{h}+h$. The slope of the straight line joining the two points of curvature change is equal to $\pm c_{0}$. Thus, which of $c \tau_{H}+H$ and $c \tau_{h}+h$ is bigger depends on whether $c$ is bigger or smaller than $c_{0}$. Again, care should be exercised as zero, $c \tau_{H}+H$ and $c \tau_{h}+h$ could be in any order-it is the number of real roots of the second auxiliary cubic equation that determines this order.

Finally, when $b>(3 / 2) a^{2} / 4$, not one of the resolvent quadratic equations has real roots. One still needs to find identifiable points on the "sub-quartic" $x^{2}\left(x^{2}+a x+b\right)$ from which "separator" lines can be drawn. There is just one such point-where the third derivative of $x^{2}\left(x^{2}+a x+b\right)$ vanishes. This point is the only root $\phi=-a / 4$ of the resolvent linear equation: $4 x+a=0$. One then draws the only available "separator"- the line $-c(x+$ $a / 4)+(1 / 16) a^{2}\left[b-(3 / 4)\left(a^{2} / 4\right)\right]$. It intersects the ordinate at the "marker" point $t=$ $-(1 / 4) a c+(1 / 16) a^{2}\left[b-(3 / 4)\left(a^{2} / 4\right)\right]$. If the signs of $a$ and $c$ are opposite, a second "separator" line, $-c x+(1 / 16) a^{2}\left[b-(3 / 4)\left(a^{2} / 4\right)\right]$ (it is parallel to the first), can provide sharper bounds on the roots (this line is not useful if $a$ and $c$ are with the same sign). Care should be exercised as one could have $t<0, t=0$, or $t>0$ - see Figures 6.1 to 6.4 in the Online Supplementary Material, on which the corresponding analysis can be found.

\section{Classification of the Roots of the Quartic Equation}

Every possible case for non-zero $a, b$, and $c$ has been thoroughly analyzed. The cases of $c$ equal to $c_{1,2}$, or $\gamma_{1,2}$, or $c_{0}$ do not get special attention-should one or more of $a, b$, and $c$ be zero or should $c$ be equal to one of the above, the analysis (not presented here) follows trivially.

The results of the investigation are presented in figures with labels $i . j$ in the Online Supplementary Material, where $i$ and $j$ are positive integers. The figures can be grouped into a (rather large) table. For ease of reference, an effort has been made to keep the individual figures independent from each other and for this purpose, each Figure $i . j$ contains a short description of the situation, analysis based on solving cubic equations, and analysis based on solving quadratic equations only.

The index $i$ in Figure $i . j$ runs from 1 to 6 and labels the rows of the table:

$\boldsymbol{i}=\mathbf{1}$ : This is the case of $b<0$. The roots $c_{1,2}, \gamma_{1,2}$, and $\rho_{1,2}$ of the first, fourth, and fifth resolvent quadratic equation, respectively, are real. The roots $\rho_{1,2}$ have opposite signs. When $i=1$, the index $j$ runs from 1 to 14 (there are fourteen columns). The first seven of these correspond to the seven possible ranges for $c$ when $a<0$; the remaining seven are the seven possible ranges for $c$ when $a>0$.

$\boldsymbol{i}=2$ : This is the case of $0<b \leq a^{2} / 4$. The roots $c_{1,2}, \gamma_{1,2}$, and $\rho_{1,2}$ of the first, fourth, and fifth resolvent quadratic equation, respectively, are again real. This time the roots $\rho_{1,2}$ have the same sign (opposite to the sign of $a$ ). When $i=2$, the index $j$ again runs from 1 to 14 (there are fourteen columns) with the first seven of these corresponding to the seven possible ranges for $c$ when $a<0$ and the remaining seven corresponding to the seven possible ranges for $c$ when $a>0$.

$\boldsymbol{i}=\mathbf{3}$ : This is the case of $a^{2} / 4<b \leq(9 / 8) a^{2} / 4$. The roots $c_{1,2}$ and $\gamma_{1,2}$ of the first and fourth resolvent quadratic equation, respectively, are real, but the roots $\rho_{1,2}$ of the fifth resolvent quadratic equation, are not real. The roots $\sigma_{h, H}$ of the sixth resolvent quadratic equation are real and these are used in the analysis. When $i=3$, the index $j$ again runs from 1 to 14 (there are fourteen columns). The first seven of these correspond to the seven 
possible ranges for $c$ when $a<0$; the remaining seven are the seven possible ranges for $c$ when $a>0$.

$\boldsymbol{i}=4$ : This is the case of $(9 / 8) a^{2} / 4<b \leq(4 / 3) a^{2} / 4$. The roots $c_{1,2}$ and $\gamma_{1,2}$ of the first and fourth resolvent quadratic equation, respectively, are real, but the roots $\rho_{1,2}$ and $\sigma_{h, H}$ of the fifth and sixth resolvent quadratic equation, respectively, are not real. The roots $\tau_{h, H}$ of the second resolvent quadratic equation are real and these are used in the analysis. When $i=4$, the index $j$ again runs from 1 to 14 (there are fourteen columns). The first seven of these correspond to the seven possible ranges for $c$ when $a<0$; the remaining seven are the seven possible ranges for $c$ when $a>0$.

$\boldsymbol{i}=5$ : This is case of $(4 / 3) a^{2} / 4<b \leq(3 / 2) a^{2} / 4$. The roots $c_{1,2}$ of the first resolvent quadratic equation are real, but the roots $\gamma_{1,2}, \rho_{1,2}$, and $\sigma_{h, H}$, of the fourth, fifth, and sixth resolvent quadratic equation, respectively, are not real. The roots $\tau_{h, H}$ of the second resolvent quadratic equation are real and these are again used in the analysis. When $i=5$, the index $j$ runs from 1 to 10 (there are ten columns). The first five of these correspond to the five possible ranges for $c$ when $a<0$; the remaining five are the five possible ranges for $c$ when $a>0$ - there are no $\gamma$ 's anymore.

$\boldsymbol{i}=\mathbf{6}$ : This is the case of $(3 / 2) a^{2} / 4<b$. Not one of the resolvent quadratic equations has real roots. But the graph of the "sub-quartic" $x^{2}\left(a x^{2}+b x+c\right)$ still has a "blemish" that can be used and extract one or two "separator" lines for the analysis. This is the point $\phi=-a / 4$ where the third derivative of $x^{2}\left(a x^{2}+b x+c\right)$ is zero. i.e. $\phi$ is the root of the resolvent linear equation $4 x+a=0$. When $i=6$, the index $j$ runs from 1 to 4 (there are only four columns): $a<0$ with $c<0, a<0$ with $c>0, a>0$ with $c<0$, and $a>0$ with $c>0$.

To find the relevant case, one has to see first in which of the above ranges the coefficient $b$ of the quadratic term falls into. The relevant row of the table is then selected by the particular value of $b$.

Next, if the sign of the coefficient $a$ of the cubic term is negative, one should look at columns 1 to 7 for the first four rows of the table, columns 1 to 5 for the fifth row and columns 1 and 2 for the sixth row of the table. If $a$ is positive, one should look at the other columns of the relevant row.

It is the place of the coefficient $c$ of the linear term within the chain of inequalities (18) that determines which particular column applies, namely, $c$ selects the individual cell in the table that is relevant.

Finally, the value of the coefficient $d$ of the free term determines, within that cell, which one of the cases labeled by lower case Roman numerals applies (either for the analysis based on cubic equations or for the analysis based on solving quadratic equations only).

As example of the above and demonstration of how to use this work, consider the quartic equation $x^{4}+2 x^{3}-4 x^{2}-13 x-3=0$ for which, obviously, one has $a>0, b<0, c<0$, and $d<0$.

Firstly, given that $b<0, a>0$, and $c<0$, one determines that Figures 1.8 to 1.11 in the Online Supplementary Material are relevant, that is, here are 4 possible cases, depending on the coefficient $c$ of the linear term.

To determine which of these 4 cases is the relevant one for the given equation, two resolvent quadratic equations should be solved first: equation (6), the roots of which are given by (7) and equation (15), the roots of which are given by (16). One will also need to solve equation (19) with roots (20) which will be needed afterwards.

Given that $a=2$ and $b=-4$, equation (6) becomes $x^{2}+10 x-656 / 27=0$ and the roots (7) are: $c_{1}=2.023$ and $c_{2}=-12.023$. Also, $c_{0}=-5$. Equation (15) becomes $x^{2}+(176 / 27) x-320 / 27=0$ and the roots $(16)$ are: $\gamma_{1}=1.481$ and $\gamma_{2}=-7.999$. Equation 
(19) becomes $x^{2}+2 x-4=0$ and the roots (20) are: $\rho_{1}=1.236$ and $\rho_{2}=-3.236$. Hence, $c \rho_{1}=-16.069$ and $c \rho_{2}=42.069$.

Clearly, one has $c=-13<c_{2}<\gamma_{2}<c_{0}<0<\gamma_{1}<c_{1}$ for this equation. Hence, Figure 1.8 in the Online Supplementary Material applies.

Given that $d<0$, one has to look at the upper half-plane of Figure 1.8 in the Online Supplementary Material. One immediately finds that $0<-d<c \rho_{2}$ and that section (iii) of the analysis based on solving quadratic equations only on Figure 1.8 in the Online Supplementary Material applies. Thus, the quartic equation $x^{4}+2 x^{3}-4 x^{2}-13 x-3=0$ has two real roots. One of them is between $\rho_{2}=-3.236$ and $-d / c=-0.2308$. The other root is greater than $\rho_{1}=1.236$. The roots of the equation are: $x_{1}=2.335, x_{2}=-0.2526$, and $x_{3,4}=-2.041 \pm 0.9605 i$. The real roots $x_{1,2}$ are exactly in their predicted isolation intervals.

\section{Outcomes, Discussion and Concluding Remarks}

When only quadratic equations are solved, the method leaves some indeterminacy as to the exact number of roots within the intervals determined by solving the resolvent quadratic equations. This residual indeterminacy can be fully lifted if one applies Arnon's complete root classification (given in the Introduction). Despite the complexity of the coefficients $p, q$, and $r$ of the depressed quartic and, in turn, the complexity of the conditions of the complete root classifications [manifested through $p, q, \delta(p, q, r)$, and $L(p, q, r)$ ], one can determine the exact number of roots of the quartic equation within each interval determined by solving the resolvent quadratic equations only.

A common outcome in both tiers of the analysis (solving cubic equations or solving quadratic equations only) is the arising need to find a bound of the biggest root from above or of the smallest root from below. For that, one needs to use a polynomial root bound. There are many existing root bounds — see [10,11,13] — and different root bounds would perform differently, depending on the coefficients of the equation. Just to name a few, one can use, for example, the Cauchy bound, which is the bigger of 1 and the sum of the absolute values of all coefficients of the monic quartic equation. Or one can use instead the stricter root bound [12]: the bigger of 1 and the sum of the absolute values of all negative coefficients of the equation. Or one can consider the bound [13]: $1+\sqrt[k]{H}$, where $k=1$ if $a<0, k=2$ if $a>0$ and $b<0$, and $k=3$ if $a>0$ and $b>0, c<0$, and $k=4$ if the only negative coefficient is $d$ (if all coefficients are positive, the upper root bound is zero). $H$ is the biggest absolute value of all negative coefficients in $x^{4}+a x^{3}+b x^{2}+c x+d$. To find the lower root bound, one needs to determine the negative of the upper root bound of $x^{4}-a x^{3}+b x^{2}-c x+d$.

The real version of the Siebeck-Marden Theorem [14-20], stated and proved by Northshield in [20], is as follows: "Any three real numbers, not all equal, are the projections of the vertices of some equilateral triangle in the plane. For a cubic polynomial $p(x)$ with three real roots (not all equal), the inscribed circle of the equilateral triangle that projects onto those roots itself projects to an interval with endpoints equal to the roots of $p^{\prime}(x)$."

Recently, by solving the Siebeck-Marden-Northshield triangle, the isolation intervals of the real roots of the symbolic cubic equation were determined in terms of the coefficients of this equation [21].

In this vein, it would be very interesting to link the proposed method to the relationship between quartic equations and tetrahedral symmetries [22] and, in particular, to the vertices 
of a tetrahedron aligned with the roots of a quartic [23] — as done in the analysis of the cubic equation in [21].

Supplementary Information The online version contains supplementary material available at https://doi. org/10.1007/s40819-021-01152-w.

Acknowledgements It is a pleasure to thank Elena Tonkova for useful discussions and Milena E. Mihaylova for the help with the figures.

Funding Open Access funding provided by the IReL Consortium

Open Access This article is licensed under a Creative Commons Attribution 4.0 International License, which permits use, sharing, adaptation, distribution and reproduction in any medium or format, as long as you give appropriate credit to the original author(s) and the source, provide a link to the Creative Commons licence, and indicate if changes were made. The images or other third party material in this article are included in the article's Creative Commons licence, unless indicated otherwise in a credit line to the material. If material is not included in the article's Creative Commons licence and your intended use is not permitted by statutory regulation or exceeds the permitted use, you will need to obtain permission directly from the copyright holder. To view a copy of this licence, visit http://creativecommons.org/licenses/by/4.0/.

\section{References}

1. Pacioli, L.: Summa de arithmetica, geometria, proportioni e proportionalitá: Distinctio IX-Tractatus XI (tractatus de computis et scripturis). Translated by Carlo Antinori, Rivista Bancaria (1959)

2. Cardano, G.: Ars Magna or the Rules of Algebra, Translated and Edited by T. Richard Witmer, Dover (2000)

3. Havelock, T.H.: Optical dispersion: a comparison of the maxima of absorption and selective reflection for certain substances. Proc. R. Soc. Sect. A Math. Phys. Sci. 84, 512-526 (1911)

4. Arnon, D.S.: Geometric reasoning with logic and algebra. Artif. Intell. 37(1-3), 37-60 (1988). https:// doi.org/10.1016/0004-3702(88)90049-5

5. Johnson, J.R.: Algorithms for polynomial real root isolation (Ph.D. thesis, 1991). In: Caviness, B.F., Johnson, J.R. (Eds.) Quantifier Elimination and Cylindrical Algebraic Decomposition. Springer (1998)

6. Yang, L., Hou, X.R., Zeng, Z.B.: Complete discrimination system for polynomials. Sci. China (Ser. E Technol. Sci.) 39(6), 628-646 (1996). https://doi.org/10.1360/ye1996-39-6-628

7. Liang, S., Jeffrey, D.J.: An algorithm for computing the complete root classification of a parametric polynomial. In: Calmet, J., Ida, T., Wang D. (Eds.) Artificial Intelligence and Symbolic Computation, Proceedings of the 8th International Conference, AISC 2006, Beijing, China, September 20-22, 2006. Springer (2006)

8. Liang, S., Jeffrey, D.J., Maza, M.M.: The complete root classification of a parametric polynomial on an interval. In: ISSAC 2008, Proceedings of the 21st International Symposium on Symbolic and Algebraic Computation, Linz/Hagenberg Austria (2010)

9. Prodanov, E.M.: On the determination of the number of positive and negative polynomial zeros and their isolation. Open Math. (De Gruyter) 18, 1387-1412 (2020). https://doi.org/10.1515/math-2020-0079. arXiv: 1901.05960

10. Rahman, Q.I., Schmeisser, G.: Analytic Theory of Polynomials. Oxford University Press, Oxford (2002)

11. Prasolov, V.V.: Polynomials. Springer, Berlin (2010)

12. Prodanov, E.M.: New bounds on the real polynomial roots. Comptes rendus de l'Academie bulgare des Sciences (to appear). arxiv:2008.11039

13. Dickson, L.E.: First course in the theory of equations. Braunworth (1922)

14. Marden, M.: Geometry of polynomials, Math. Surveys no. 3, American Mathematical Society, Providence, RI (1966)

15. Marden, M.: A note on the zeros of the sections of a partial fraction. Bull. Am. Math. Soc. 51, 935-940 (1945). https://doi.org/10.1090/S0002-9904-1945-08470-5

16. Siebeck, J.: Ueber eine neue analytische behandlungweise der brennpunkte. J. Reine Angew. Math. 64, $175-182(1864)$

17. Kalman, D.: An elementary proof of Marden's theorem. Am. Math. Month. 115(4), 330-338 (2008). https://doi.org/10.1080/00029890.2008.11920532 
18. Badertscher, E.: A simple direct proof of Marden's theorem. Am. Math. Monthly 121(6), 547-548 (2014). https://doi.org/10.4169/amer.math.monthly.121.06.547

19. Steiner, J.: Géométrie pure. Développment d'une série de théorèmes relatifs aux sections coniques. Ann. Math. Pures Appl. 19, 37-64 (1828/1829)

20. Northshield, S.: Geometry of cubic polynomials. Math. Mag. 86(20), 136-143 (2013). https://doi.org/10. 4169/math.mag.86.2.136

21. Prodanov, E.M.: The Siebeck-Marden-Northshield theorem and the real roots of the symbolic cubic equation, arXiv:2107.01847

22. Chalkley, R.: Quartic equations and tetrahedral symmetries. Math. Mag. 48(4), 211-215 (1975). https:// doi.org/10.1080/0025570X.1975.11976489

23. Nickalls, R.W.D.: The quartic equation: alignment with an equivalent tetrahedron. Math. Gazette 96, 49-55 (2012). https://doi.org/10.1017/S0025557200003958

Publisher's Note Springer Nature remains neutral with regard to jurisdictional claims in published maps and institutional affiliations. 